\title{
A case of ulcerative colitis presenting with cerebral venous thrombosis
}

\author{
Junghwan Lee ${ }^{1,2}$, Sung Wook Hwang ${ }^{1}$, Jinhee Lee ${ }^{2}$, Kyung Hwa Jung ${ }^{2}$, Ha Il Kim², Sang Hyoung Park ${ }^{1}$, \\ Dong-Hoon Yang ${ }^{1}$, Byong Duk Ye ${ }^{1}$, Jeong-Sik Byeon ${ }^{1}$, Seung-Jae Myung ${ }^{1}$, Suk-Kyun Yang ${ }^{1}$ \\ Departments of ${ }^{1}$ Gastroenterology and ${ }^{2}$ Internal Medicine, Asan Medical Center, University of Ulsan College of Medicine, Seoul, Korea
}

Patients with inflammatory bowel disease (IBD) have been reported to have an increased risk of thromboembolism. Cerebral venous thrombosis (CVT) is a rare but serious extraintestinal manifestation of IBD. Due to its highly variable manifestation and low incidence, CVT is not usually readily recognized by physicians. Herein, we report a case of a 35-year-old male presenting with CVT associated with ulcerative colitis (UC). The patient was admitted with chief complaints of bloody diarrhea that had started 3 days prior. Sigmoidoscopy showed hyperemic and edematous mucosa, friability, and shallow ulcers from the sigmoid colon to the rectum suggestive of IBD. Three days later, the patient started complaining of a headache, and gradually developed a decreased level of consciousness. Magnetic resonance imaging of the brain revealed CVT with hemorrhagic infarctions. An angiogram was obtained to evaluate the extent of CVT, and anticoagulation therapy was initiated with intravenous heparin. During hospitalization, he was diagnosed with UC and treated with 5-aminosalicylic acid. After discharge, the patient was recovered without neurological deficit, and remission of UC was also obtained. The presence of headache or acute worsening of neurological status in a patient with IBD should alert the health professionals about the possibility of CVT. (Intest Res 2018;16:306-311)

Key Words: Inflammatory bowel disease; Colitis, ulcerative; Cerebral venous thrombosis; Complications; Thromboembolism

\section{INTRODUCTION}

Inflammatory bowel disease (IBD) is a systemic disease that can be accompanied by various extraintestinal manifestations. Of these manifestations, thromboembolism, such as deep venous thrombosis and pulmonary embolism are well recognized. ${ }^{1}$ Cerebral venous thrombosis (CVT) is a fatal extraintestinal manifestation of IBD that is very rarely reported in Korea and worldwide. ${ }^{2}$ The presentation of patients with CVT may be variable. The most common clinical manifestations are headaches, vomiting, seizures, focal neurologic deficit, decreased level of consciousness and visual impair-

Received June 29, 2017. Revised August 6, 2017.

Accepted August 15, 2017. Published online September 29, 2017

Correspondence to Sung Wook Hwang, Department of Gastroenterology,

Asan Medical Center, University of Ulsan College of Medicine, 88 Olympic-ro

43-gil, Songpa-gu, Seoul 05505, Korea. Tel: +82-2-3010-3195, Fax: +82-2-

476-0824,E-mail: snow903@gmail.com ment. However, unspecified headache may be the one and only presenting symptom. ${ }^{3,4}$ Due to its nonspecific manifestation and low incidence, CVT is not readily recognized and occasionally misdiagnosed by physicians. Herein, we report a case of a 35-year-old male presenting with CVT associated with UC that was successfully treated with anticoagulation and 5-aminosalicylic acid.

\section{CASE REPORT}

A 35-year-old male was admitted with chief complaints of bloody diarrhea and mild fever for 3 days. The patient had been suffering from watery diarrhea and general weakness for 2 months. The patient was not on any medication nor had any other medical history in the past. Laboratory findings showed an elevated white blood cell counts $(14,600 /$ $\mu \mathrm{L})$, ESR ( $42 \mathrm{~mm} / \mathrm{hr})$, CRP level $(2.82 \mathrm{mg} / \mathrm{dL})$, and D-dimer $(7.8 \mu \mathrm{g} / \mathrm{mL})$, and a decreased hemoglobin level $(8.6 \mathrm{~g} / \mathrm{dL})$,

( ) Copyright 2018. Korean Association for the Study of Intestinal Diseases. All rights reserved.

This is an Open Access article distributed under the terms of the Creative Commons Attribution Non-Commercial License (http://creativecommons.org/licenses/by-nc/4.0)

which permits unrestricted non-commercial use, distribution, and reproduction in any medium, provided the original work is properly cited. 
and platelet count $(100,000 / \mu \mathrm{L})$. All other biochemical, electrolyte tests were within the normal limits. Sigmoidoscopic findings showed hyperemic and edematous mucosa, friability, and shallow ulcers from the sigmoid colon to the rectum (Fig. 1). With suspicion of UC, he was provided supportive care and intravenous antibiotics while waiting for serologic and pathologic results. Three days later, the patient started complaining of a headache and gradually developed a decreased level of consciousness and intermittent dystonic posture of the right arm. On neurologic examination, slight motor weakness in the right upper and lower limbs was detected. There were no obvious pathologic reflexes. A CT scan of the head showed hemorrhagic infarction in the left basal ganglia and infarctions in the right anterior frontal and left parietal lobe, which are outside the boundaries for an arterial territory (Fig. 2). With suspicion of venous thrombosis, MRI of the brain with venogram was obtained, and diffuse dural venous thrombosis with multiple hemorrhagic infarctions was identified (Fig. 3). Doppler ultrasonography of lower extremities showed no deep venous thrombosis. The patient was immediately transferred to the intensive care unit, and anticoagulation therapy was initiated with intravenous heparin infusion. An angiogram was obtained to evaluate the extent of CVT, but invasive endovascular therapy was not performed due to concerns of complications (Fig. 4). Sigmoidoscopic biopsies showed chronic active colitis with ulcer, crypt distortion, and cryptitis, which was consistent with UC. Perinuclear antineutrophil cytoplasmic antibody was positive and fecal calprotectin was highly elevated $(6,545 \mu \mathrm{g} / \mathrm{g})$. UC was diagnosed based on clinical history, laboratory studies, and biopsies of the colon. The patient was treated with a combination of oral and rectal 5-aminosalicylic acid. A prothrombotic workup that assessed for protein C, protein $\mathrm{S}$, and antithrombin III deficiencies, anticardiolipin antibody, and fasting homocysteine was negative, except for lupus anticoagulant (LA). His initial Mayo score was 11, but symptoms of UC and CVT were gradually improved during hospitalization. When he was discharged 4 weeks later, the score improved to partial Mayo score 1. After discharge, the patient was recovered without neurologic deficit, and remission of UC (Mayo score 0) was also obtained (Fig. 5). Anticoagulation was continued with warfarin, and follow-
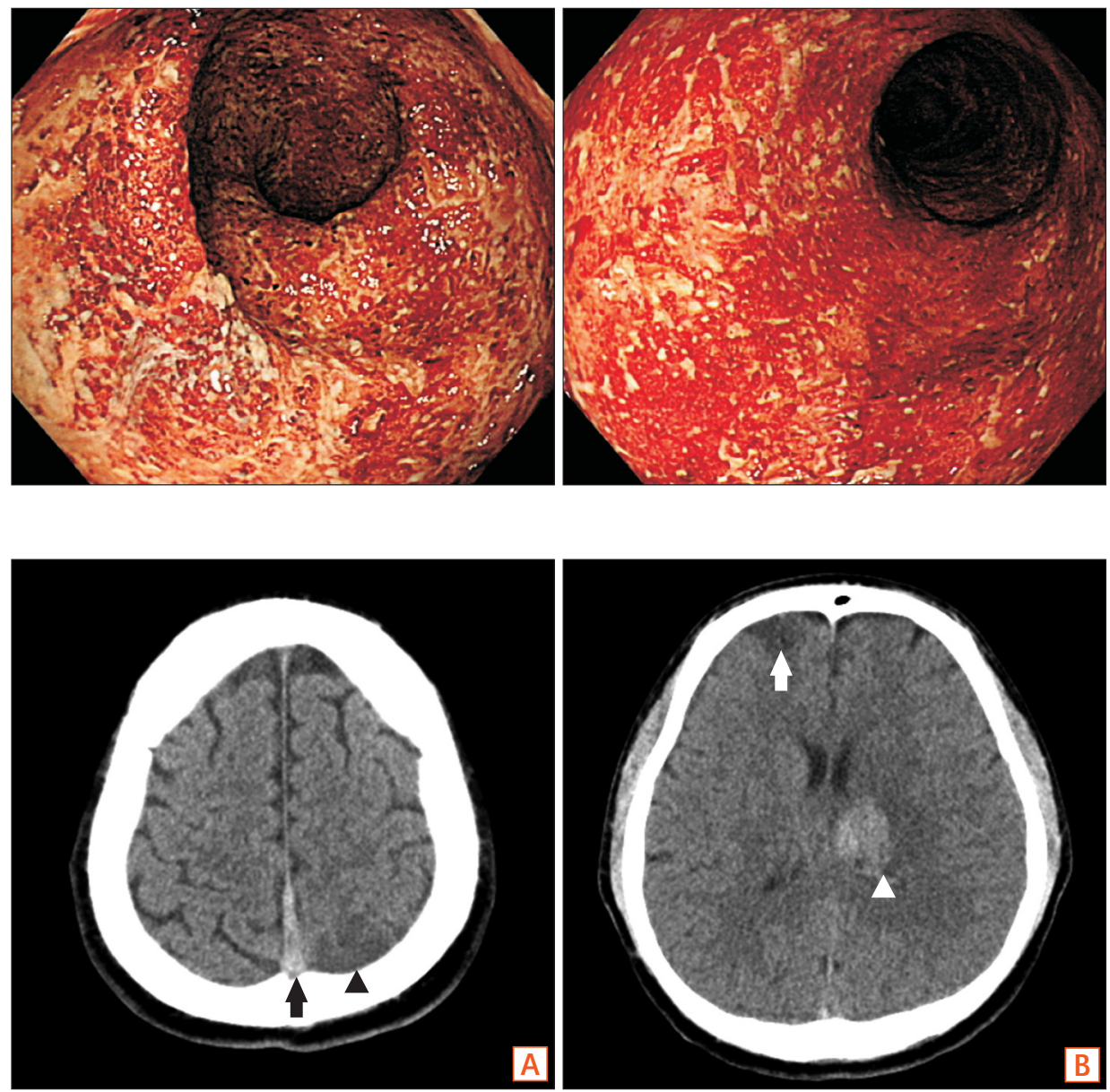

Fig. 1. Endoscopic findings. Endoscopic findings show hyperemic and edematous mucosa, friability, and shallow ulcers from the sigmoid colon to the rectum.

Fig. 2. Non-enhance brain CT findings. (A) The CT shows hyperattenuating thrombus in the superior sagittal sinus (black arrow) with hypoattenuating lesion suggesting infarction at the left parietal lobe (black arrowhead), (B) and right frontal lobe (white arrow), as well as hemorrhagic infarction in left basal ganglia (white arrowhead). 

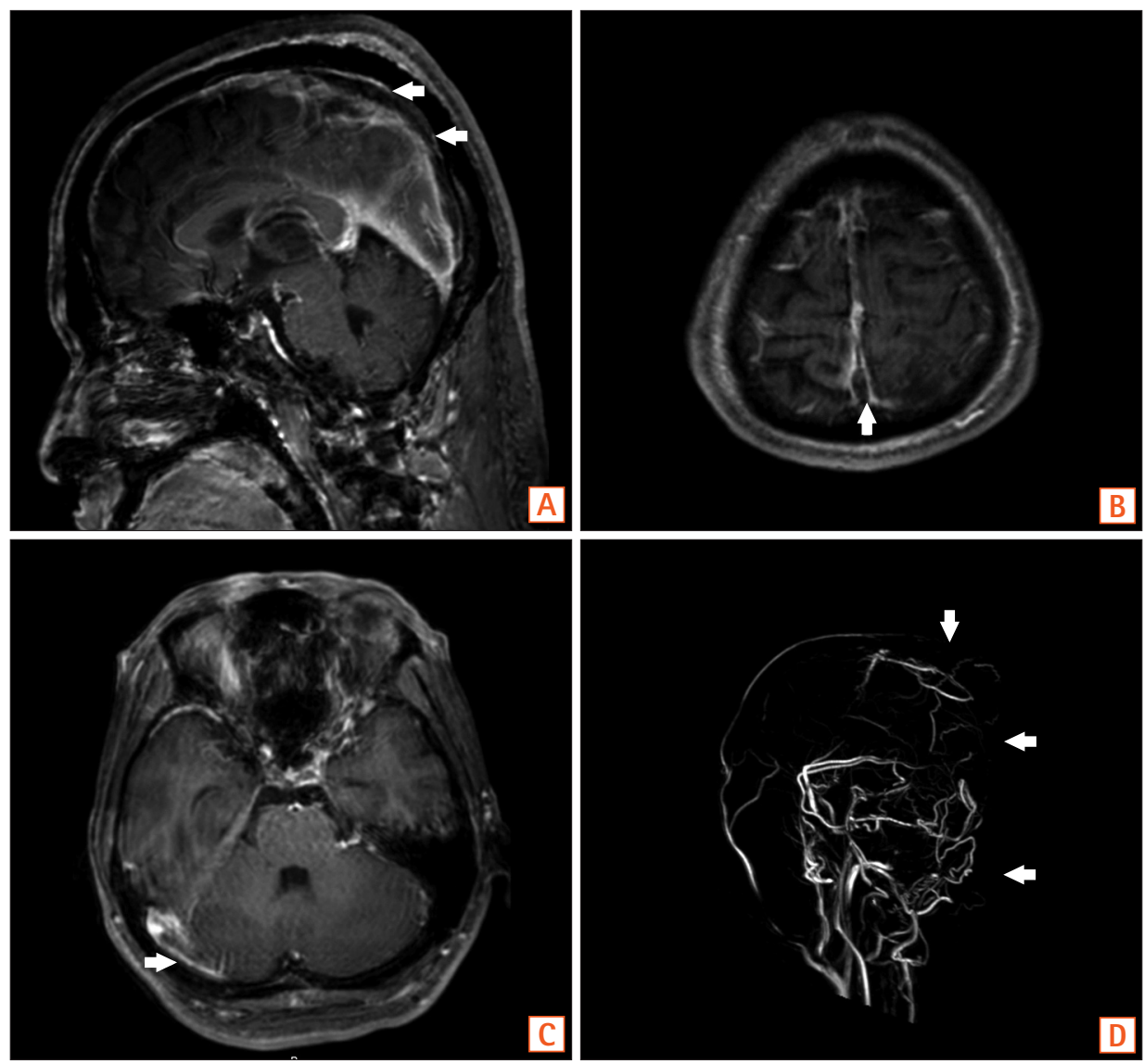

Fig. 3. Brain MRI with venography. MRI with venography shows venous thrombosis in the superior sagittal sinus $(A, B, D$, arrows) and, the right transverse sinus ( $C$, arrow).
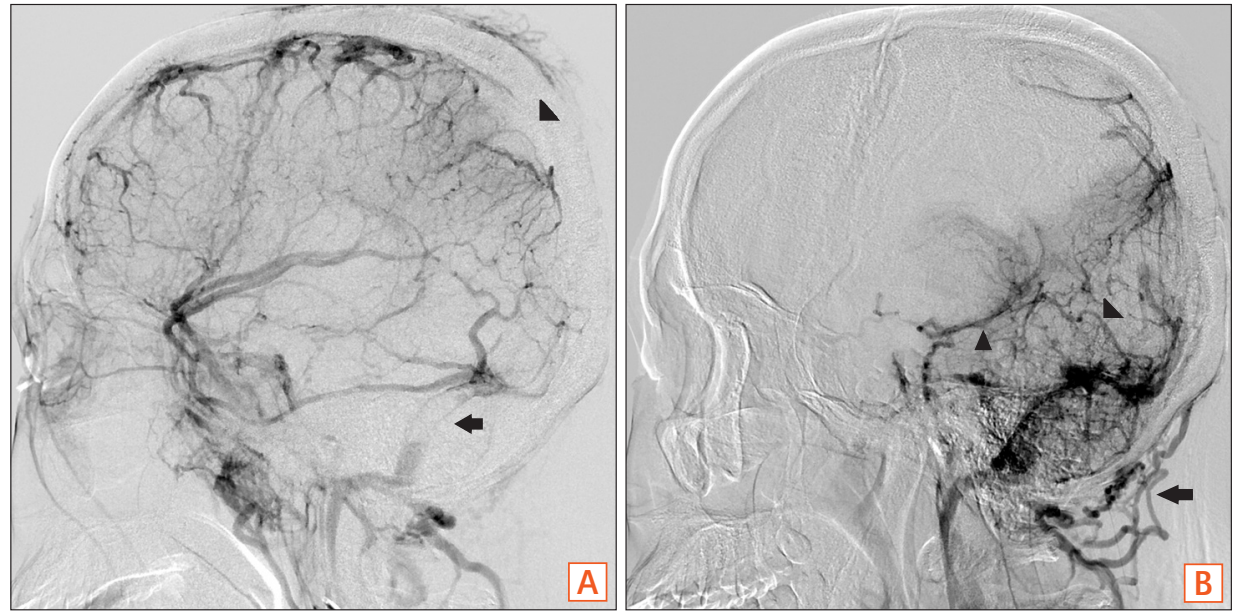

Fig. 4. Interventional angiography. Interventional angiogram shows partial occlusion of the superior sagittal sinus $(A$, arrowhead) and straight sinus ( $B$, arrowhead) with asymmetrical filling of the left side of the deep vein ( $B$, arrowhead) and both transverse sinuses ( $A$, arrow) with collateral venous drainage via the posterior neck venous channel ( $B$, arrow).

up imaging after 8 weeks showed a decreased extent of CVT (Fig. 6). Persistently positive LA was detected 12 weeks apart, and mild to moderate thrombocytopenia (platelet count, $50,000-100,000 / \mu \mathrm{L}$ ) persisted without bone marrow abnormalities.

\section{DISCUSSION}

IBD, especially at the time of disease flares, is a risk factor for venous thromboembolism (VTE), which is often a serious event associated with high mortality. ${ }^{5}$ Inflammatory status confers various hemostatic alteration, and mucosal inflammation has been proposed to be responsible for the 
hypercoagulable state in patients with IBD. ${ }^{6}$

CVT is a relatively rare cause of stroke that affects young individuals (under the age of 50). ${ }^{7} \mathrm{CVT}$ is also a rare extraintestinal manifestation of IBD. The mean age of IBD patients at the time of the CVT is significantly younger when compared with CVT patients without IBD; in the reviewed case reports, the mean age of IBD patients at the time of the CVT was $<29$ years. ${ }^{4.8}$ Previous case reports have shown that CVT appears to be more common in UC than CD, and a female predominance has also been noticed. ${ }^{4}$ Additionally, anemia and thrombocytosis have been suggested as significant risk factors for CVT. ${ }^{4,9}$ Coagulation abnormalities (increased fibrinogen, elevated factor VII, elevated factor VIII, antithrombin III deficiency, and protein $\mathrm{C}$ and protein $\mathrm{S}$ deficiency) often coexist with IBD and return to normal values when the

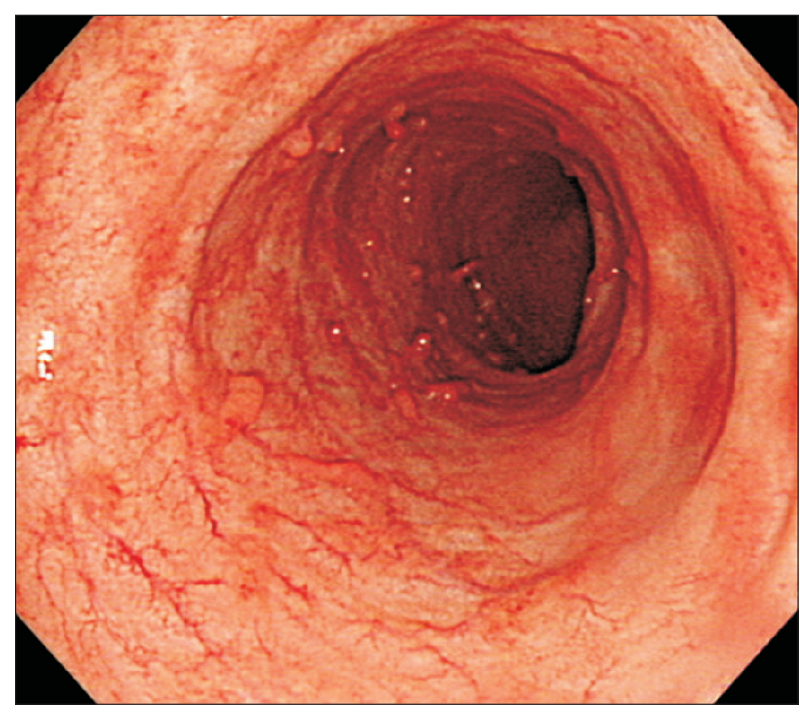

Fig. 5. Endoscopic findings. Follow-up endoscopy shows endoscopic remission of UC. intestinal inflammation is controlled. Antiphospholipid antibodies (aPLs) were also present in some IBD patients with CVT. ${ }^{10}$ In this case study, our patient was a 35 -year-old male with anemia who was positive for aPLs

The variable presentation of CVT and its insidious course often contribute to delays in diagnosis. Headache is the most common and sometimes only presenting symptom, which makes it a clinical challenge to diagnosis $\mathrm{CVT}^{3,11}$ Further increased intracranial hypertension may cause vomiting, diplopia, visual impairment, and decreased level of consciousness. Focal neurologic symptoms may suggest the presence of a venous infarction and hemorrhagic conversion, which has been reported in more than one-third of patients. ${ }^{8}$ In this case, the patient initially complained of a headache only, which progressed to a decreased level of consciousness and focal neurologic deficit. A high index of suspicion is required to diagnosis CVT.

The high D-dimer level may suggest an acute and extensive CVT. However, a low level of D-dimer does not rule out CVT, since the D-dimer levels decline with time from the onset of symptoms. ${ }^{12}$ In this case, the D-dimer $(7.8 \mu \mathrm{g} / \mathrm{mL})$ level was elevated, which implied the possibility of a thromboembolic event. aPLs are a prothrombotic group of antibodies found in a variety of chronic inflammatory diseases and are used as diagnostic laboratory markers for antiphospholipid antibody syndrome. In previous studies, the presence of aPLs was reported in IBD patients, but the clinical significance was unclear. ${ }^{4}$ This patient showed persistent positive antiphospholipid antibody in the form of LA 12 weeks apart, and he showed unexplained thrombocytopenia rather than thrombocytosis. These findings may suggest the possible co-presence of antiphospholipid syndrome, which makes our case even rarer. Although a recent cohort study demonstrated the presence of aPLs was not associated with a risk
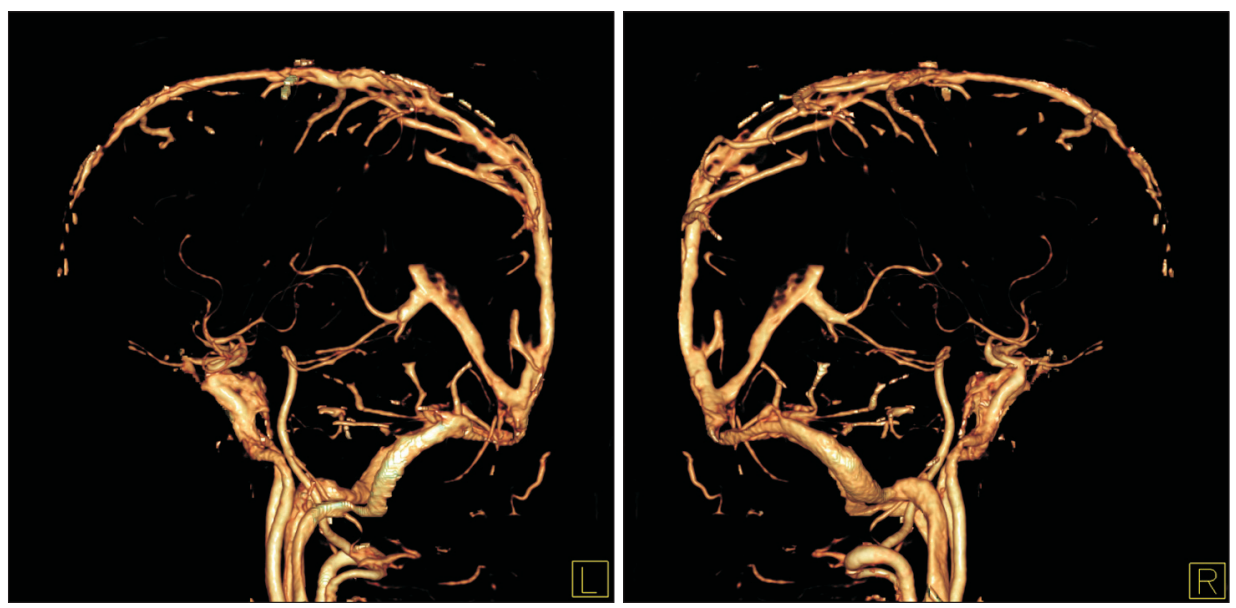

Fig. 6. CT venogram. CT venogram shows a decreased extent of multiple filling defects in the superior sagittal sinus, straight sinus, transverse sinuses and sigmoid sinus. $L$ left; $R$, right. 
of venous thrombotic events in CD patients, aPLs may have certain pathogenetic roles in thromboembolic disease. ${ }^{13}$ Further study seems to be required to determine a pathogenetic role of aPLs in IBD patients with thromboembolic complications.

Non-contrast head CT, which is the most frequently performed imaging study for the evaluation of patients with neurological deficit, has poor sensitivity to detect $\mathrm{CVT} .^{12} \mathrm{IBD}$ patients with an intracerebral hemorrhage or infarctions outside the boundaries for an arterial territory should be searched for underlying CVT. ${ }^{14} \mathrm{MRI} /$ magnetic resonance venogram is recommended as a preferred brain image to diagnose CVT. ${ }^{15}$ The most commonly affected venous sinus is the superior sagittal (62\%); however, multiple cerebral thrombosis has been reported in half of the patients, as in

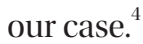

Anticoagulation treatment is the standard treatment for patients with CVT. Anticoagulation prevents further thrombus growth and facilitates recanalization. ${ }^{3}$ Previous studies revealed anticoagulation treatment was associated with a lower RR of death in patients with CVT. ${ }^{16}$ Therefore, the current guidelines suggest that patients with CVT should be treated with low molecular weight heparin or intravenous heparin, even in the presence of intracranial hemorrhage. ${ }^{15}$ Although there is a great concern about the hemorrhagic complications (e.g., hemorrhagic infarctions, cerebral hemorrhages, and intestinal bleeding), the risks should be carefully weighted and anticoagulation treatment should be considered. Previous studies have shown that IBD patients with CVT who did not receive anticoagulation treatment showed a poorer outcome compared to those who received it. ${ }^{4,17}$ In our case, the patient was treated with 5 -aminosalicylic acid, and anticoagulation was initiated with intravenous heparin and continued with warfarin. Although patients with multiple cerebral thrombosis and hemorrhagic infarction in the basal ganglia have been reported to have a higher risk of mortality, ${ }^{8}$ our patient was recovered without neurologic deficit. In this case, CVT may not only be associated with UC but possibly also with antiphospholipid syndrome. Followup imaging after 8 weeks also showed decreased but residual thrombus. Therefore, we continued anticoagulation therapy and considered infinite administration of warfarin as was previously recommended in a study by Katsanos et al. ${ }^{4}$ A multidisciplinary approach (gastroenterology, neurology, rheumatology and hematology) will be used to care for this patient and decide the treatment duration. Disease activity of UC will also be considered in the course of anticoagulation therapy.
Regarding hospitalized UC patients, prophylactic anticoagulation is recommended in Western countries, but the clinical effectiveness of prophylactic anticoagulation in Asians needs to be further studied. ${ }^{2}$ In this case, UC was suspected initially, but the definite diagnosis of UC was confirmed after the diagnosis of CVT. Therefore, anticoagulation was started after the diagnosis of CVT for therapeutic purpose.

Corticosteroid treatment in IBD patients with CVT is still up for debate. Although some previous studies considered that steroid treatment does not increase the risk for VTE, but rather promote anti-inflammatory effects and decrease hypercoagulability in IBD patients, other studies reported highdose steroid treatment is associated with an increased risk of developing VTE, especially in patients with other risk factors (e.g., inherited coagulopathies, oral contraceptive use). ${ }^{18-20}$ Our patient was treated without corticosteroids out of concern for a possible adverse effect of corticosteroids.

In conclusion, the presence of a severe headache or neurologic signs or symptoms in a patient with IBD should prompt clinicians to suspect the possibility of CVT, which may be the presenting symptom for a newly diagnosed active phase of IBD.

\section{FINANCIAL SUPPORT}

The authors received no financial support for the research, authorship, and/or publication of this article.

\section{CONFLICT OF INTEREST}

No potential conflict of interest relevant to this article was reported.

\section{AUTHOR CONTRIBUTION}

J.L., S.W.H., and J.L. wrote the manuscript. K.H.J. and H.I.K helped manuscript and figure corrections.

S.H.P., D.H.Y., B.D.Y., J.S.B., S.J.M., and S.K.Y. provided a critical revision of the manuscript.

All authors contributed to writing this case report and were responsible for final approval of the manuscript.

\section{REFERENCES}

1. Bernstein CN, Blanchard JF, Houston DS, Wajda A. The incidence of deep venous thrombosis and pulmonary embolism among patients with inflammatory bowel disease: a population-based cohort study. Thromb Haemost 2001;85:430-434. 
2. Cho YH, Chae MK, Cha JM, et al. Cerebral venous thrombosis in a patient with Crohn's disease. Intest Res 2016;14:96-101.

3. Bushnell C, Saposnik G. Evaluation and management of cerebral venous thrombosis. Continuum (Minneap Minn) 2014;20:335-351.

4. Katsanos AH, Katsanos KH, Kosmidou M, Giannopoulos S, Kyritsis AP, Tsianos EV. Cerebral sinus venous thrombosis in inflammatory bowel diseases. QJM 2013;106:401-413.

5. Nguyen GC, Sam J. Rising prevalence of venous thromboembolism and its impact on mortality among hospitalized inflammatory bowel disease patients. Am J Gastroenterol 2008;103:22722280.

6. Danese S, Papa A, Saibeni S, Repici A, Malesci A, Vecchi M. Inflammation and coagulation in inflammatory bowel disease: the clot thickens. Am J Gastroenterol 2007;102:174-186.

7. Bousser MG, Ferro JM. Cerebral venous thrombosis: an update. Lancet Neurol 2007;6:162-170.

8. Ferro JM, Canhão P, Stam J, Bousser MG, Barinagarrementeria F; ISCVT Investigators. Prognosis of cerebral vein and dural sinus thrombosis: results of the International Study on Cerebral Vein and Dural Sinus Thrombosis (ISCVT). Stroke 2004;35:664670.

9. Chauhan S, Sachdev A, D'Cruz S, Jain K, Singh R. Cerebral venous thrombosis in a patient with ulcerative colitis. Inflamm Bowel Dis 2007;13:1588-1589.

10. Kawanishi M, Yoshida Y, Sakaguchi I, Nagano F, Kato K, Miyake H. Cerebral venous sinus thrombosis in a patient with ulcerative colitis. J Stroke Cerebrovasc Dis 2003;12:271-275.

11. Crassard I, Bousser MG. Headache in patients with cerebral venous thrombosis. Rev Neurol (Paris) 2005;161:706-708.
12. Saposnik G, Barinagarrementeria F, Brown RD Jr, et al. Diagnosis and management of cerebral venous thrombosis: a statement for healthcare professionals from the American Heart Association/American Stroke Association. Stroke 2011;42:11581192.

13. Sipeki N, Davida L, Palyu E, et al. Prevalence, significance and predictive value of antiphospholipid antibodies in Crohn's disease. World J Gastroenterol 2015;21:6952-6964.

14. Wasay M, Azeemuddin M. Neuroimaging of cerebral venous thrombosis. J Neuroimaging 2005;15:118-128.

15. Einhäupl K, Stam J, Bousser MG, et al. EFNS guideline on the treatment of cerebral venous and sinus thrombosis in adult patients. Eur J Neurol 2010;17:1229-1235.

16. de Bruijn SF, Stam J. Randomized, placebo-controlled trial of anticoagulant treatment with low-molecular-weight heparin for cerebral sinus thrombosis. Stroke 1999;30:484-488.

17. Nudelman RJ, Rosen DG, Rouah E, Verstovsek G. Cerebral sinus thrombosis: a fatal neurological complication of ulcerative colitis. Patholog Res Int 2010;2010:132754. doi: 10.4061/2010/132754.

18. Markowitz RL, Ment LR, Gryboski JD. Cerebral thromboembolic disease in pediatric and adult inflammatory bowel disease: case report and review of the literature. J Pediatr Gastroenterol Nutr 1989;8:413-420.

19. Stolz E, Klötzsch C, Schlachetzki F, Rahimi A. High-dose corticosteroid treatment is associated with an increased risk of developing cerebral venous thrombosis. Eur Neurol 2003;49:247248.

20. Richard S, Fairise A, Lacour JC, Ducrocq X. Cerebral venous thrombosis in inflammatory bowel diseases. Inflamm Bowel Dis 2010;16:366-367. 